Kompass

Ophthalmologie

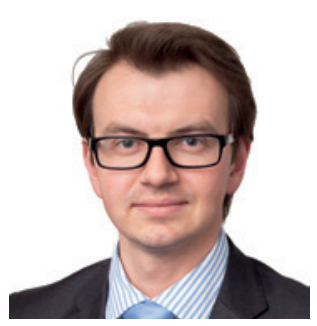

\section{Matus Rehak}

Klinik und Poliklinik für Augenheilkunde, Universitätsklinikum Leipzig,

Leipzig, Deutschland

Nicht nur die Menschen beeinflussen und verändern die Umwelt, sondern die Umwelt hat auch einen starken Einfluss auf die Menschen. Natürlich gilt das auch für unsere Gesundheit, und etliche Augenerkrankungen zeigen eine enge Korrelation mit dem Lebensstil. Über die Wirkung der Tagesbeleuchtung auf die Entwicklung von Myopie bei Kindern wurde viel erforscht und Präventionskonzepte in einigen Ländern reflektieren diese Erkenntnisse. Somit versucht man die Zeit, die die Kinder draußen im Freien verbringen, zu verlängern, z.B. in den Schulpausen oder indem Teile des Unterrichts ins Freie verlagert werden. Auch im erwachsenen Alter beeinflussen Lebensstil oder Beruf die Einwicklung von einigen Augenerkrankungen. Das Trockene Auge ist nur ein Beispiel davon.

Seit langer Zeit wissen wir, dass Computertätigkeit und trockene Luft in klimatisierten Räumlichkeiten einen starken Einfluss auf die Symptome beim Sicca-Syndrom haben. Daher sind die Ergebnisse der Studie der Kollegen aus Mexiko zwar nicht überraschend, trotzdem sind sie aber sehr interessant. Hernandez-Llamas und Kollegen verglichen die Häufigkeit der Sicca-Sympto-

Kompass Ophthalmol 2021;7:1

DOI: 10.1159/000514864

\title{
Umwelteinflüsse und das Auge
}

matik bei Arbeitern, die zwar überwiegend im Freien tätig sind, aber oft eine starke Exposition gegenüber Staub und vielen anderen chemischen Stoffe (z.B. auf der Baustelle) haben und bei Arbeitern mit einer klassischen Büro-Tätigkeit am PC und überwiegend in klimatisierten Räumen. Die Ergebnisse belegen, dass die Büroarbeiter deutlich häufiger über Symptome des Trockenen Auges klagen.

Auf die Problematik des Sicca-Syndroms fokussiert in diesem Heft auch der Wissenstransfer, der von Herrn Professor Bachmann zum Thema Diquafosol fürs Trockene Auge verfasst wurde.

Ein weiteres Thema, dem wir uns in der aktuellen Ausgabe von Kompass Ophthalmologie widmen, ist die COVID-19-Infektion. Seit einem Jahr leben wir in der Pandemie, sehr viel wurde über die Übertragung, Behandlung und Prävention gesagt und geschrieben. Mit zunehmender Zahl der Patienten, die eine COVID-19-Infektion überstanden haben, häufen sich derzeit die Berichte über unterschiedliche Organmanifestationen der Komplikationen nach einer durchgemachten SARS-CoV-2-Erkrankung. Es ist bekannt, dass diese Infektion nicht nur eine klassische Atemwegserkrankung ist, sondern dass das Virus auch Endothelzellen und Nervengewebe angreift. Die Kollegen aus Hannover beschreiben den Fall einer jungen Patientin, die nach einer COVID19-Erkrankung mit einem milden Verlauf Myasthenia gravis mit binokularen Doppelbildern entwickelte. Diese sehr schön informativ verfasste Kasuistik dokumentiert, wie vielfältig und weitreichend die Folgen der SARS-CoV-2-Erkrankung sein können. Auf das Thema der COVID-19-Infektion fokussiert auch der Beitrag von Herrn Professor Rohrschneider, der über die ambulante Behandlung von Glaukompatienten in diesen Zeiten berichtet.

Ich wünsche Ihnen eine spannende und informative Lektüre in diesen besonderen Zeiten der Pandemie.

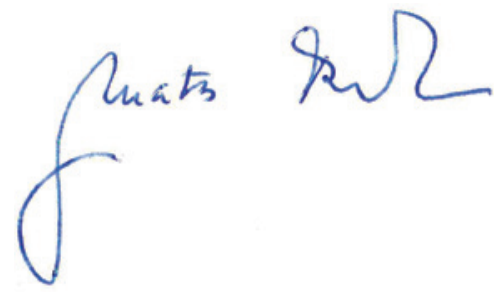

Prof. Dr. Matus Rehak 